\title{
Wireless Power Generation Strategy Using EAP Actuated Energy Harvester for Marine Information Acquisition
}

\author{
So-Nam Yun ${ }^{1}$, Eun-A Jeong ${ }^{1}$, Hwang-Hun Jeong ${ }^{1}$, Ky-Yun Lee ${ }^{2}$ \\ ${ }^{1}$ Energy Plant Research Division, Korea Institute of Machinery \& Materials, Daejeon, Korea \\ ${ }^{2}$ Department of Polymer Science and Engineering, Chungnam National University, Daejeon, Korea \\ E-mail:ysn688@kimm.re.kr,kylee@cnu.ac.kr \\ Received July 25, 2011; revised August 21, 2011; accepted September 6, 2011
}

\begin{abstract}
The energy of a radio wave is reduced through the dispersion, the refraction and the absorption because the medium transferring the vibration of a radio wave is the seawater. In the end the reduced energy of a radio wave causes the reduced transmitting length for communication, the long postponed communication and the frequent error. The subsea communication for the marine environment monitoring which must overcome the weak points of the RF wave and the most practical method for the marine sensor network realization is to use the acoustic wave method, but the energy consumption rate of the acoustic wave communication method is about 100 times greater than the one of the RF wave method. So, the power supply of the sensor node in the marine sensor network system is the most important interest field. In this study, the sample which consists of an acrylic elastomer (VHB4905 film from $3 \mathrm{M}$ ), conductible carbon grease(from MG chemical) and electric wire for the basic study of an energy harvesting strategy and technique using EAP actuator was fabricated, and the conductible carbon grease was used for an electrode. The characteristics of the fabricated sample were analyzed through the experiment. We also mixed carbon grease with aluminum powder for conductibility improvement, and the effect of the mixed electrode was confirmed through the conductivity measurement.
\end{abstract}

Keywords: Electroactive Polymer, Energy Harvester, Electric Conductivity, Wireless Power generation, Marine Information Acquisition

\section{Introduction}

The monitoring strategy of the marine environment using WSN (Wireless Sensor Network) with a battery or an energy scavenging system is the core technology for the security of the marine resources, the military information acquisition and the data insurance for the weather prediction [1-3].

However, the sensor network technology of the top side using the conventional type RF (Radio Frequency) is difficult to apply to subsea because the medium of the seawater is different from the air one. The energy of a radio wave is reduced through the dispersion, the refraction and the absorption because the medium transferring the vibration of a radio wave is the seawater. In the end the reduced energy of a radio wave causes the reduced transmitting length for communication, the long postponed communication and the frequent error. The subsea communication for the marine environment monitoring which must overcome the weak points of the RF wave and the most practical method for the marine sensor network realization is to use the acoustic wave method, but the energy consumption rate of the acoustic wave communication method is about 100 times greater than the one of the RF wave method. So, the power supply of the sensor node in the marine sensor network system is the most important interest field.

In case of the sensor node which is installed at the inshore and offshore, the power supply to the sensor node is operated by the electrical wire. In case of the sensor node installed at the subsea, all of them have batteries and are retrieved when the batteries are discharged using the buoy, AUV (Automatic under Vehicle) or UUV (Unmanned Underwater Vehicle). The cost for the sensor withdraws and for the battery change is greater than the sensor cost itself when the cost of the sensor installed at the subsea is low. As a result, the low cost sensors which are died of natural causes are thrown away. If the self 
energy generating and the supply method for the sensor power supply are developed, the realization and the operation of the marine sensor network will be easy, and this kind of system has the environmental and the economical advantages [4].

There are two kinds of the vibration energy harvesting method from material point of view. One is the PZT (Lead-Zirconate-Titanate) actuator method, and the other is EAP actuator method. The wake-up circuit, power management circuit, rectifier and MOSFET (Metal-Oxide Semiconductor Field Effect Transistor) have been researched because the harvested energy quantity from the vibration energy is very low for the material limitation and structure characteristics [5-9].

In this study, the sample which consists of a acrylic elastomer (VHB4905 film from $3 \mathrm{M}$ ), conductible carbon grease(from MG chemical) and electric wire for the basic study of an energy harvesting strategy and technique using EAP actuator was fabricated, and the conductible carbon grease was used for an electrode. The characteristics of the fabricated sample were analyzed through the experiment. We also mixed carbon grease with aluminum powder for conductibility improvement, and the effect of the mixed electrode was confirmed through the conductivity measurement which is derived from the output voltage and the output current from the fabricated sample with a mixed electrode.

\section{Experimental Apparatus}

\subsection{Principal of Energy Harvesting}

Figure 1 shows the principal of the electrical energy production when the conductible polymer is used for the energy generation. The energy density, $u$, of the electric field when Maxwell stress is not considered is expressed as Equation (1).

$$
u=\frac{1}{2} \varepsilon \varepsilon_{0} E^{2}
$$

where, $\varepsilon$ means the relative permittivity and $\varepsilon_{0}$ is the permittivity $\left(8.85 \times 10^{-12} \mathrm{~F} / \mathrm{m}\right)$ in the free space. $E$ $[\mathrm{V} / \mathrm{m}]$ is the electric field.

The electrostatic capacity, $C$ [C], which is derived by the conductible polymer from the Figure $\mathbf{1}$ is described as Equation (2).

$$
C=\frac{\varepsilon \varepsilon_{0} A}{Z}
$$

where, $z$ is the length between two electrodes, $A\left[\mathrm{~m}^{2}\right]$ is the cross-sectional area between electrodes. And the electric field $E$ is expressed as Equation (3).

$$
E=\frac{V}{z}
$$

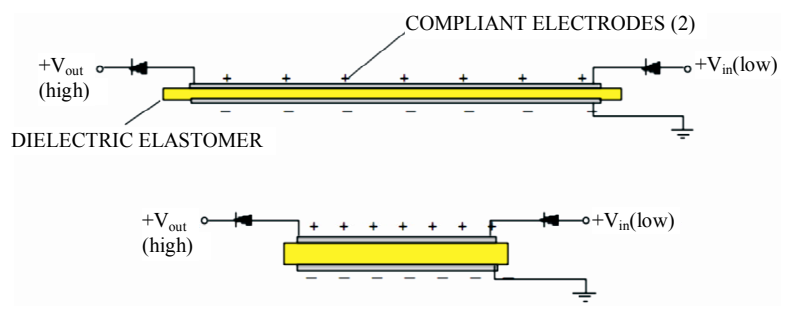

Figure 1. Energy generated from dielectric elastomer when a force is impressed on compliant electrodes.

where, $V[\mathrm{~V}]$ means the voltage drop which is generated by the electrostatic capacity.

The outputted energy, $U$ [W], from the conductible polymer is as Equation (4).

$$
U=u A z
$$

We can get the final equation Equation (5) substituting Equation (1) and Equation (3) for Equation (4).

$$
U=\frac{1}{2} C V^{2}
$$

From the Equation (5), we can know that the energy is outputted in accordance with the electrostatic capacity change of the conductible polymer $[9,10]$.

Figure 1 explains the energy generation state by the electrostatic capacity change which is also governed by the real external force. The electrostatic capacity is varied if the length between the electrodes and the crosssectional area of the electrode are changed because the electrostatic capacity is in inverse proportion to the surface area of the polymer actuator and is also in proportion to the length between the electrodes.

\subsection{Experimental Setup and Procedure}

Many kinds of EAP materials such as HS3 silicone, Polyurethanes, Silicone NuSil CF 19-2186 and Acrylic Elastomer are used for the energy harvesting mechanism. In this study, the Acrylic Elastomer has been selected for the energy harvesting material because this material has characteristics such as a long displacement, a high operating force and a high density.

In this study, the Acrylic Elastomer of VHB 4905 film from $3 \mathrm{M}$ was selected [11], and the electrode of the conductible carbon grease from MG chemical was used for the enough transformation for the flexible Acrylic Elastomer [12].

Figure 2 shows the photo of the energy harvesting device which is the laboratory-made prototype and consists of the Acrylic Elastomers, carbon greases and the electric wires. The made energy harvester is two layered experimental model. An Acrylic Elastomer was displaced at the center position and the carbon grease with an electrode was painted as a same height of $5 \mathrm{~mm}$ to the both side of 


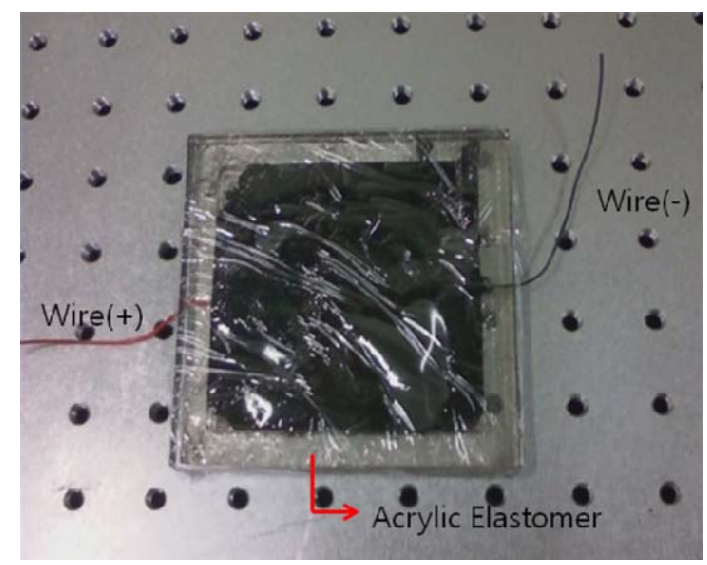

Figure 2. A fabricated energy harvester using EAP.

the Acrylic Elastomer. Finally two wires were equipped at the experimental model for measurement the outputted power. Above-mentioned experimental model was made on the plate which is the transparent acryl and the size is $100 \mathrm{~mm}$ (length) $\times 100 \mathrm{~mm}$ (width) $\times 5 \mathrm{~mm}$ (height)

Figure 3 shows the photo of the experimental setup for scavenging the vibration energy. An oscilloscope (DSO3230A, Agilent) for measurement the output power of the energy scavenging equipment like Figure $\mathbf{2}$ and a notebook for measured data storage have been equipped. In this study, the energy harvesting strategy for confirmation the conductivity variation by impurity ratio change of the Aluminum powder which is mixed to the electrode of the carbon grease was conducted. The mixed ratios of the Aluminum powder to the carbon grease were $0 \mathrm{wt} \%, 5 \mathrm{wt} \%$ and $10 \mathrm{wt} \%$, respectably.

The mass of $75.5 \mathrm{~g}$ for the objectivity guarantee of the experimental condition for the energy harvesting capability estimation was used and the mass was dropped from the height of $40 \mathrm{~mm}$. Finally the outputted voltage and current were measured by the power meter and the energy harvesting ratio by the mixed Aluminum powder variation is calculated. Total energy quantity which was

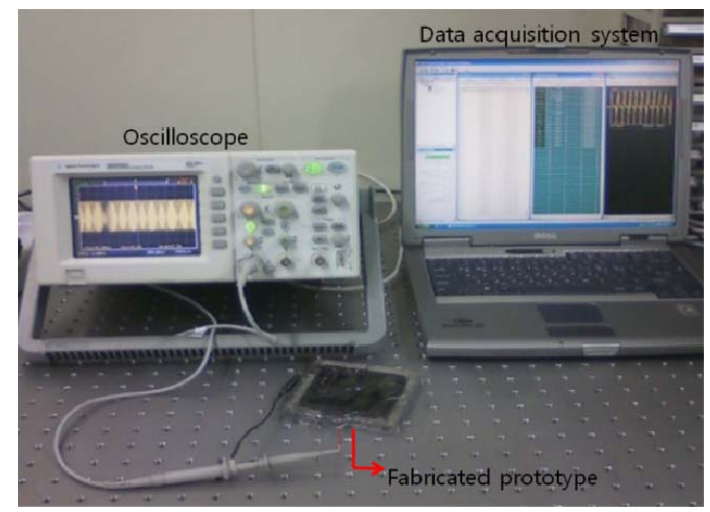

Figure 3. Experimental setup for confirmation the energy harvest validity. gotten from the vibration energy was calculated by numerical integration of the output voltage and current during $6 \mathrm{~s}$.

\section{Experimental Results and Discussion}

Figures 4, 5 and 6 show the output voltage characteristics of the manufactured energy harvester of which electrode is carbon grease and have Aluminum impurity of $0 \%, 5 \%$ and $10 \%$, respectably. And the mass for shock energy generation to the fabricated energy harvester is $75.5 \mathrm{~g}$ and the dropped height is $40 \mathrm{~mm}$.

The sampling speed is $2.0 \mathrm{kSa} / \mathrm{s}$. Also, $\mathrm{x}$-axis is divided by $50 \mathrm{~ms} / \mathrm{div}$. and y-axis is divided by $200 \mathrm{mv} / \mathrm{div}$. The fluctuated part in the graph means the contact timing between the mass and the test object system. We can know from the output voltage result graphs that that the more Aluminum impurity is induced the higher voltage output.

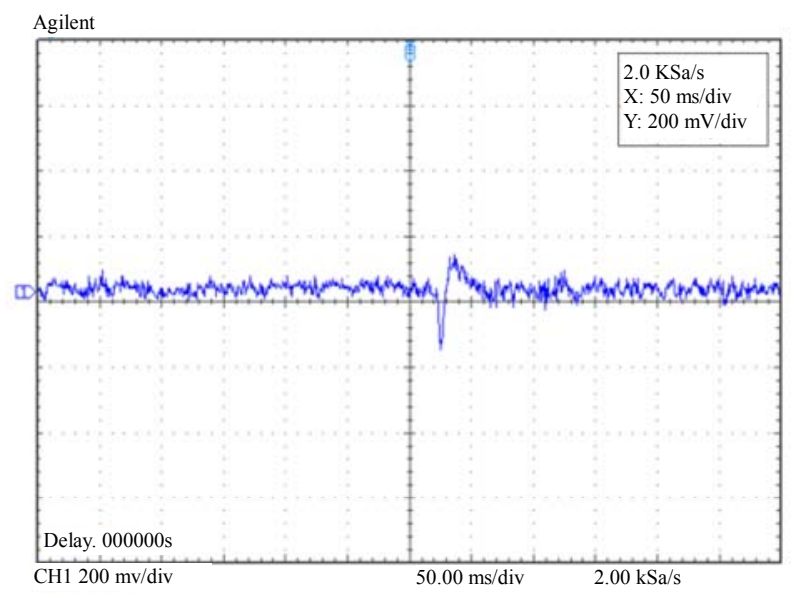

Figure 4. Output voltage from energy harvester without impurity.

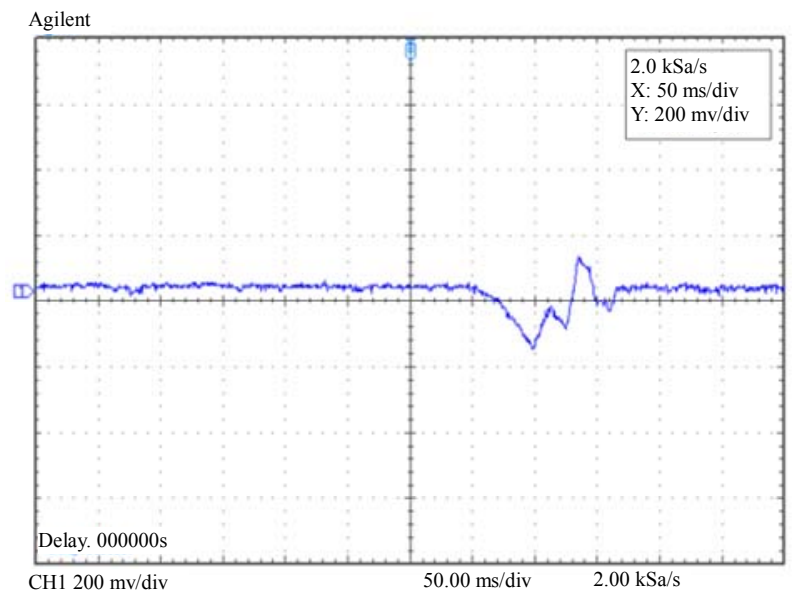

Figure 5. Output voltage from energy harvester with $\mathbf{5 \%}$ Aluminum impurity. 


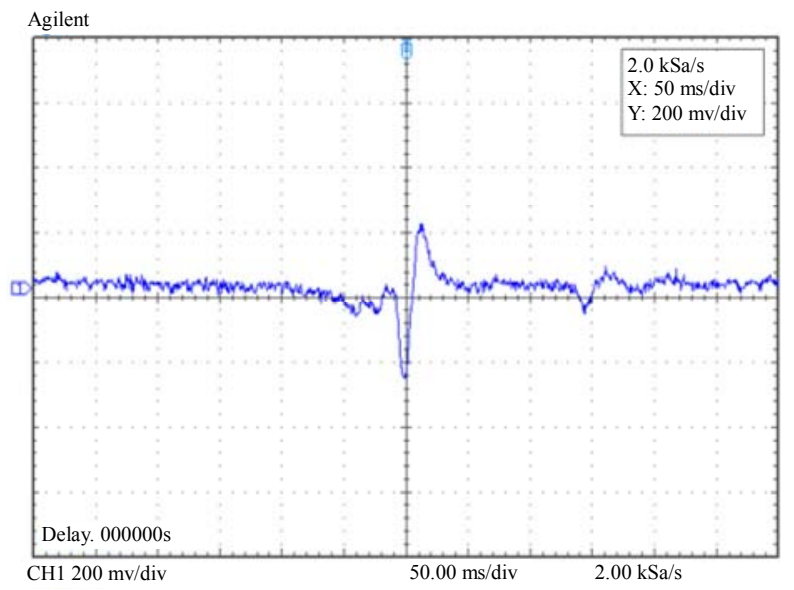

Figure 6. Output voltage from energy harvester with $10 \%$ Aluminum impurity.

Figures 7, 8 and 9 show the voltage characteristics but these contain the current information because these results were measured using the resistance of $10 \mathrm{M} \Omega$ inside the energy harvester. And also $\mathrm{x}$-axis scale is 50 $\mathrm{ms} / \mathrm{div}$. and y-axis scale $200 \mathrm{mv} / \mathrm{div}$.

The electric output power is calculated by the numerical integration of the experimental results from Figure 4 to Figure 8 and the product of integrated voltage and current. And we can also compare the harvested-quantity by the impurity ratio. The oscilloscope's sampling speed is $2 \mathrm{kSa} / \mathrm{s}$ and the sampled data were 1200 . The experiments were operated many times by control system with an oscilloscope. And Figure 4 to Figure 8 are the representative output results. (6).

The numerical integration was conducted by Equation

$$
S_{\mathrm{sum}}=\sum_{i=1}^{1199}\left(\frac{(s(i+1)+s(i))}{2} \Delta t\right)
$$

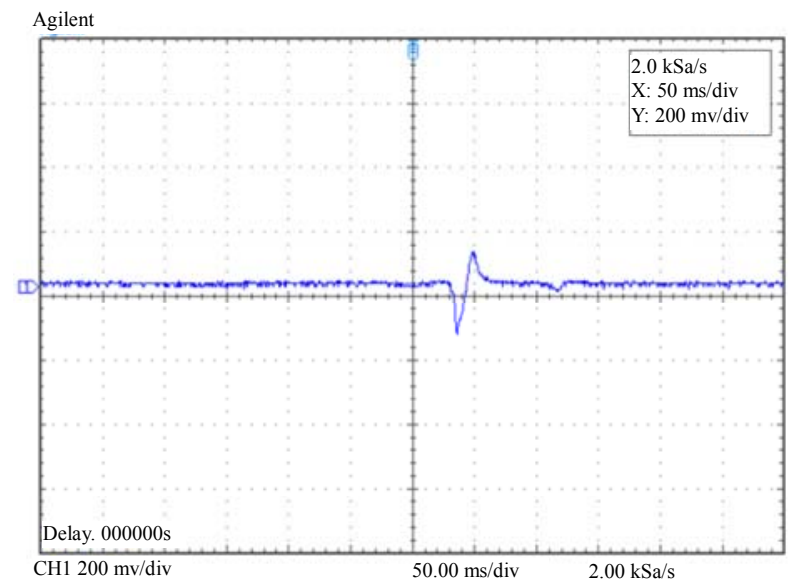

Figure 7. Output current from energy harvester without Aluminum impurity.

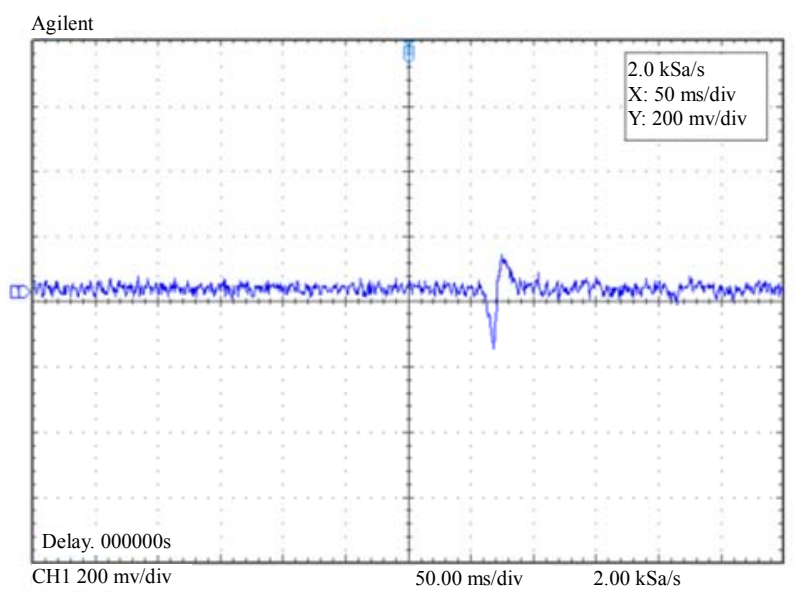

Figure 8. Output current from energy harvester with 5\% Aluminum impurity.

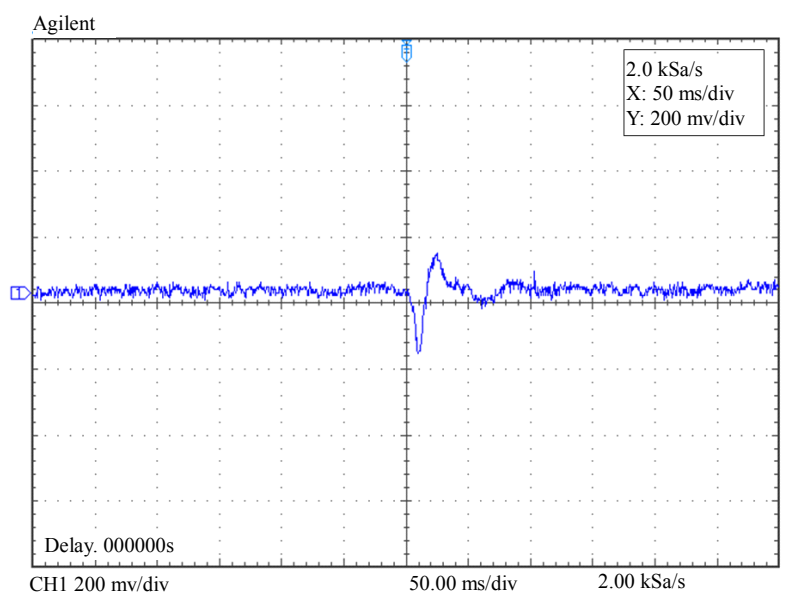

Figure 9. Output current from energy harvester with $\mathbf{1 0 \%}$ Aluminum impurity.

where, $S_{\text {sum }}$ means the voltage or the current value derived from the energy harvester during $6 \mathrm{~s}$.

$S_{i}(i=1,2, \cdots, 1200)$ means the data from the voltage or the current signal matrix.

Table 1 and Figure 10 show the maximum voltage, the maximum current and the maximum energy respectably from the energy harvester which composed with the acrylic elastomer and electrodes with Aluminum impurity. We can know from the Table 1 and Figure 10 that there is an Aluminum impurity effect because the

Table 1. Experimental results by vibration energy.

\begin{tabular}{cccc}
\hline & $\begin{array}{c}\text { Maxium } \\
\text { Voltage [V] }\end{array}$ & $\begin{array}{c}\text { Maxium } \\
\text { Current [mA] }\end{array}$ & $\begin{array}{c}\text { Power } \\
{[\mathrm{W} \cdot \mathrm{s}]}\end{array}$ \\
\hline $0 \% \mathrm{wt}$ & 0.147 & 0.343 & 0.0024 \\
$5 \% \mathrm{wt}$ & 0.140 & 0.097 & 0.0065 \\
$10 \% \mathrm{wt}$ & 0.230 & 0.153 & 0.0078 \\
\hline
\end{tabular}




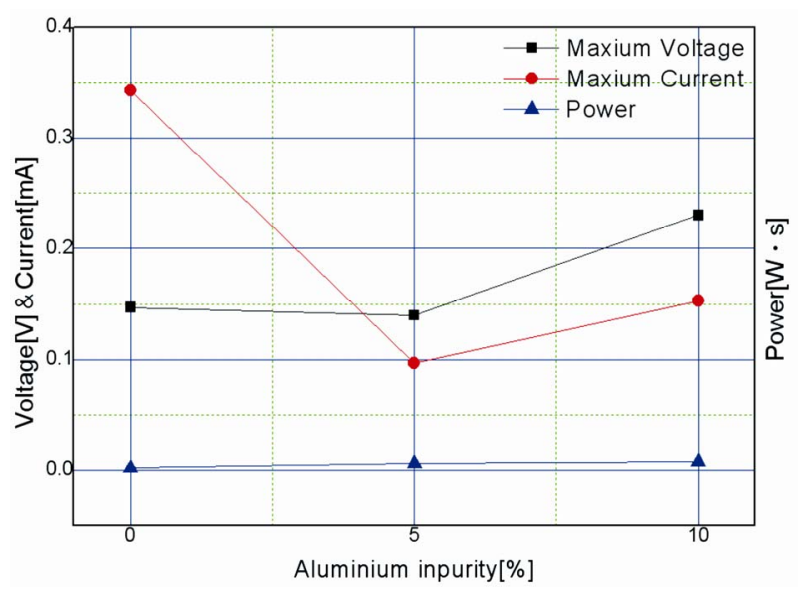

Figure 10. Output power results by Aluminum impurity ratio variation.

more Aluminum impurity is mixed to the electrode, the more harvested energy is gotten.

\section{Conclusions}

The purpose of this study is to understand the characteristics of the energy harvesting devices using vibration for marine information acquisition with a wireless sensor network system.

In this study, the sample which consists of a acrylic elastomer(VHB4905 film from $3 \mathrm{M}$ ), conductible carbon grease(from MG chemical) and electric wire for the basic study of an energy harvesting strategy and technique using EAP actuator was fabricated, and the conductible carbon grease was used for an electrode. The validity of the Aluminum impurity ratio in the main electrode was experimented and analyzed. The proposed model can be used to predict performance and to provide insights for improving the designs of energy harvesting system. It will also be useful in the design and analysis of control systems that utilize this in high performance application of vibration.

\section{References}

[1] S. H. Han and B. K. Cho, "A Study on the Development of Marine Detector Using Nano-technology," Journal of the Korean Society of Marine Environment \& Safety, Vol. 14, No. 1, 2008, pp. 39-43.

[2] H. Y. Choi and S. H. Park, "A Group Base Sub Sink Underwater Wireless Sensor Network Routing," 2006 Annual Spring Conference of the Korean Society for Simulation, 2006, pp. 149-156.

[3] Y. H. Yu, Y. S. Gang and W. B. Lee, "Development of a Floating Buoy for Monitoring Ocean Environment," Journal of the Korean Society of Marine Engineering, Vol. 33, No. 5, 2009, pp. 705-712.

[4] S. J. Park, S. H. Park, S. G. Kim and C. H. Kim, "Marine Sensor Network Technique," Journal of the Korean Institute of Information Scientists and Engineers, Vol. 28, No. 7, 2010, pp. 79-88.

[5] D. W. Kim, et al., "The Study of Improvement Performance Techniques for Self Sustaining Sensor Node System Using Acquire Vibration Energy," 2009 Annual Fall conference of the Korean Institute of Electrical Engineers, 2009, pp. 97-98.

[6] K. J. Park, et al., "Self-Powered Wireless Sensor using MEMS/MOSFET Hybrid Switch," 2010 Annual Summer Conference of the Korean Institute of Electrical Engineers, 2010, pp. 1179-1180.

[7] S. M. Kang, et al., "Power Management Circuit for Solar Cell Powered Wireless Sensor Nodes," 2009 Annual Summer Conference of the Korean Institute of Electrical Engineers, 2009, pp. 1925-1926.

[8] H. K. Chang, D. J. Kim and J. Y. Park, "Use of Piezoelectric Effect in Portable Loadless Wind-Power Source for Ubiquitous Sensor Networks," Journal of the Korean Society of Mechanical Engineers B, Vol. 34, No. 6, 2010, pp. 623-628. doi:10.3795/KSME-B.2010.34.6.623

[9] R. Perlin, et al., "High-Speed Electrically Actuated Elastomers with Strain Greater than 100\%," Journal of Science, Vol. 287, No. 5454, 2000, pp. 836-839.

[10] G. Kofod, "Dielectric Elastomer Actuators," The Technical University of Denmark, Lyngby, 2001, pp. 2-20.

[11] http://www.mgchemicals.com.

[12] http://products3.3m.com. 